\title{
A light and period study of SV Camelopardalis ${ }^{\star}$
}

\author{
B. Albayrak ${ }^{1}$, O. Demircan ${ }^{2}$, G. Djurašević ${ }^{3}$, S. Erkapić ${ }^{3}$, and H. Ak ${ }^{1}$ \\ 1 Ankara University, Science Faculty, Dept. of Astronomy and Space Sciences, 06100 Tandoğan, Ankara, Turkey \\ 2 Çanakkale Onsekiz Mart University, Faculty of Arts and Sciences, Dept. of Physics, 17100 Çanakkale, Turkey \\ 3 Astronomical Observatory, Volgina 7, 11050 Belgrade, Yugoslavia
}

Received 17 May 2001 / Accepted 22 June 2001

\begin{abstract}
New $B V R$ light curves and times of minimum light for the short period RS CVn system SV Cam were analysed to derive the physical parameters of the system and the parameters of the third body orbit. The light curves obtained at the TÜBİTAK National Observatory during two nights in 2000 show considerable asymmetry and night-to-night variations. The analysis of the light curves is made using Djurasevic's inverse problem method. The Roche model with spotted areas on the hotter primary component yields a good fit to observations. The extensive series of published photoelectric minima times indicate that the eclipsing pair orbits around the common mass center of the triple system with a period of $41.32 \mathrm{yr}$.
\end{abstract}

Key words. stars: binaries: eclipsing - stars: individual: SV Cam

\section{Introduction}

The short-period $(P \simeq 0$ d59) RS CVn type totally eclipsing binary system SV Cam $\left(V_{\max }=9{ }^{\mathrm{m}} 34, \mathrm{HD} 44982\right.$, $\mathrm{BD}+82^{\circ} 174$, HIC 32015$)$ has been extensively observed since its discovery by Guthnick (1929). Light variations and asymmetry in the overall shape of the light curves, as much as $0.1 \mathrm{mag}$ in time scales as short as one month, had been noticed by all observers (e.g. van Woerden 1957; Hilditch et al. 1979; Patkos 1982a,b; Cellino et al. 1985; Milano et al. 1986). Hilditch et al. reported that the cool secondary component is the source of distortion in the light curves. On the contrary, Cellino et al. claimed that the active spotted component should be the hotter primary. This hypothesis was supported by the brightening they observed at phase 0.5 , at the same time as the increased reddening at the primary eclipse due to the passage of the spotted area through phase 0.0. On the other hand, Özeren et al. (2001) found an excess emission in both $\mathrm{H}_{\alpha}$ and $\mathrm{H}_{\beta}$ lines connected with the secondary, cooler component of the system.

The light curve solutions indicate that the primary component is very close to its Roche limit and provides at least $90 \%$ of the total light of the system. It is therefore not

\footnotetext{
Send offprint requests to: B. Albayrak, e-mail: albayrak@astro1.science.ankara.edu.tr

* Table 1 is only available in electronic form at the CDS via anonymous ftp to cdsarc.u-strasbg.fr (130.79.128.5) or via

http://cdsweb.u-strasbg.fr/cgi-bin/qcat?J/A+A/376/158
}

possible for the fainter cool secondary (which contributes less than $10 \%$ to the system light) to be the source of large distortion in the light curves.

The spectral type of the component stars were estimated to be G2-3 V and K4 V by Hilditch et al. (1979), giving a mass ratio of about 0.71 . The later $\mathrm{G} 8 \mathrm{~V}$ classification of the primary component by Popper (1996) is based on the Na D lines. From a radial velocity study, Pojmański (1998) estimated a new mass ratio of $q=m_{\mathrm{c}} / m_{\mathrm{h}}=0.56$ and found an earlier spectral type for the primary. The indices $(\mathrm{h}, \mathrm{c})$ refer to the hotter (more-massive) primary and cooler (less-massive) secondary component respectively. From the stellar masses, he derived spectral types F5 V and $\mathrm{KO} \mathrm{V}$ for the components of SV Cam system.

The light-time effect possibility of orbital motion about a third body was first suggested by Friebos-Conde \& Herczeg (1973) to explain the period variation of SV Cam. The existence of the third body in the system was later confirmed by Hilditch et al. (1979), Cellino et al. (1985), Sarma et al. (1985), and Albayrak et al. (1999) with extended data. However, the parameters of the third body orbit were found to be very different in each study.

This paper presents three new light curves in $B, V$, and $R$, obtained in 2000. These were analysed with a fixed revised mass ratio of $q=m_{\mathrm{c}} / m_{\mathrm{h}}=0.56$. We used two different hypotheses concerning the temperature of the hotter primary component: $T_{\mathrm{h}}=6440 \mathrm{~K}$ (Hyp. I.) and $T_{\mathrm{h}}=6200 \mathrm{~K}$ (Hip. II.) corresponding to F5 V and F8 V stars, and assumed cool spots on the hotter component. 
The period variation of the system was also studied again by using the newly extended data in the hope of finding more reliable parameters of the third body orbit.

\section{New observations}

New observations of SV Cam in $B V R$ were obtained at the TÜBITAK National Observatory (Turkey) on the nights of 22 January and 2 February 2000, by using a SSP-5A photometer attached to a $0.4 \mathrm{~m}$ Cassegrain telescope. The same comparison star $\mathrm{BD}+82^{\circ} 0176$ was chosen as Milano et al. (1986). A total of 242 observations were secured in each filter. The nightly extinction coefficients for each colour were determined by using the observations of the comparison star. The instrumental differential $B, V$, and $R$ magnitudes in the sense variable minus comparison corrected for the atmospheric extinction, are given in Table 1 (accessible in electronic form). The differential $B, V$, and $R$ light and $B-V$ and $V-R$ colour curves are shown on the upper right-hand of Fig. 2. The photometric phases of the light and colour curves are calculated with the following ephemeris given by Pojmański (1998):

MinI $=$ HJD 2449350.3037+0.593071 E.

\section{Third body orbit}

During the observations three primary and two secondary minima were obtained. Their timings were calculated by using the well-known method of Kwee \& van Woerden (1956). The photoelectric minima published before mid1997 were collected by Albayrak et al. (1999). We added minima obtained later by Agerer \& Hübscher (1998), Pribulla et al. $(1999,2001)$ and by the present study. Altogether 152 primary and 18 secondary photoelectric minima are given in Table 2. Since the secondary minima are very shallow, the precise determination of the time of each minimum is strongly affected by the lightcurve asymmetry. Thus, four secondary minima times were omitted as their scatter suggests they are not as well determined as the rest of the values. They are marked in

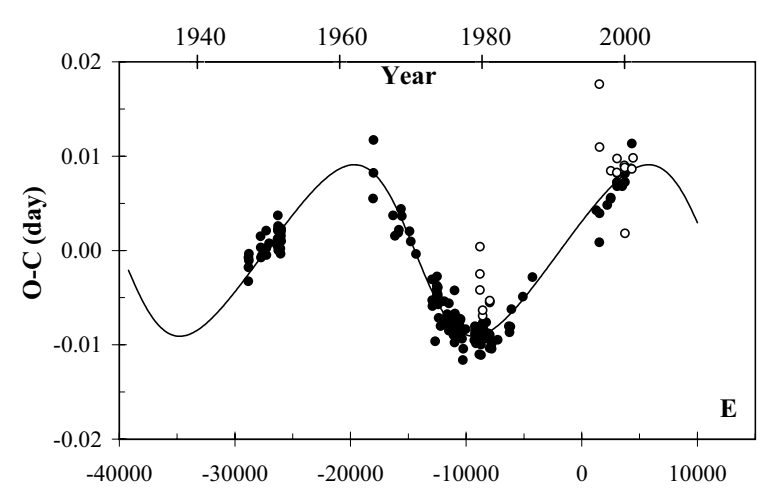

Fig. 1. The best-fitting theoretical curve superimposed on the observational $(\mathrm{O}-\mathrm{C})$ data of SV Cam (filled circles are photoelectric primary minima while open circles are photoelectric secondary minima times). italics in Table 2. The linear light elements given by Eq. (1) were used in estimating $\mathrm{C}$ values. The $(\mathrm{O}-\mathrm{C})$ diagrame in Fig. 1 can be represented by a continuous oscillatory variation covering one minimum and almost two maxima by the present observational data. This oscillation is produced by the light time effect due to a third body in the system.

To derive the light-time orbit and the parameters of the invisible component, the analysis was performed by using Mayer's (1990) equations. We used the scientific graphic, curve fitting and statistic program PRISIM (Cass 2000) to obtain the parameters, listed in Table 3 with their standard deviations. Table 3 also compares the results of this analysis with those of different authors for SV Cam. The mass of the third body may be evaluated from the mass function as the $(\mathrm{O}-\mathrm{C})$ analysis allows us to obtain $f\left(M_{3}\right)$. But, it depends on the inclination of the longperiod orbit. The inclination $i$ of the third body orbit was assumed to be equal to the inclination of the eclipsing binary orbit found as 89.6 in this study (see Table 4). Since masses of the components of the eclipsing pair were derived by us are 1.42 and $0.80 M_{\odot}$ (see Table 4$)$, the mass of the third component is $0.26 \pm 0.01 M_{\odot}$. If we take its mass and the period as $0.26 M_{\odot}$ and $41.32 \mathrm{yr}$, respectively, Kepler's third law gives the semi-major axis of the orbit to be 16.18 AU. Assuming the Hipparcos distance of SV Cam $d=84.96 \mathrm{pc}$, we get the angular separation of the third body from the eclipsing pair as $0^{\prime \prime} 19$.

\section{Light curve analysis}

To analyse the asymmetric light curves formed by the new observations, we used Djurašević's (1992a) programme, which is based on the Roche model and the principles from the paper by Wilson \& Devinney (1971). The lightcurve analysis was made by applying the inverse-problem method (Djurašević 1992b) based on Marquardt's (1963) algorithm.

The stellar size in the model is described by the filling factors for the critical Roche lobes $F_{1,2}$ of the primary and secondary components, respectively, which tell us to what degree the stars in the system fill their corresponding critical lobes. For synchronous rotation of the components these factors are expressed as the ratio of the stellar polar radii, $R_{1,2}$, and the corresponding polar radii of the critical Roche lobes, i.e., $F_{1,2}=R_{1,2} / R_{\text {Roche }_{1,2}}$. To achieve more reliable estimates of the model parameters in the light-curve analysis programme, we applied a quite dense coordinate grid, having $72 \times 144=10368$ individual elementary cells per star. The intensity and angular distribution of the radiation of individual cells are determined by the stellar effective temperature, limb-darkening, gravitydarkening and by the reflection effect are in the system.

The asymmetries in the light curves are assumed to be formed by cool spots on the hotter more massive component. In the code the spotted regions are approximated by circular spots, characterised by the temperature contrast of the spot with respect to the surrounding photosphere 
Table 2. The photoelectric minima times of SV Cam in HJD*(HJD - 2400 000).

\begin{tabular}{|c|c|c|c|c|c|c|c|c|c|c|c|}
\hline $\begin{array}{l}\text { HJD }^{*} \\
\text { primary }\end{array}$ & Ref. & HJD $^{*}$ & Ref. & HJD* $^{*}$ & Ref. & HJD $^{*}$ & Ref. & HJD $^{*}$ & Ref. & HJD* $^{*}$ & Ref. \\
\hline 32253.8190 & 1 & 33791.6582 & 1 & 41835.4800 & 9 & 42634.3500 & 9 & 43880.3930 & 9 & 45043.4053 & 18 \\
\hline 32265.6819 & 1 & 33803.5200 & 1 & 41905.4670 & 9 & 42771.3500 & 11 & 43892.2540 & 15 & 45627.5824 & 19 \\
\hline 32268.6483 & 1 & 33844.4420 & 1 & 41930.3770 & 9 & 42777.2786 & 11 & 43927.2450 & 9 & 45658.4215 & 19 \\
\hline 32281.6963 & 1 & 33895.4451 & 1 & 41931.5635 & 9 & 42777.2792 & 11 & 43928.4300 & 9 & 45696.3787 & 19 \\
\hline 32287.6263 & 1 & 33911.4575 & 1 & 41933.3437 & 9 & 42829.4700 & 9 & 44049.4184 & 9 & 45741.4540 & 19 \\
\hline 32878.9214 & 1 & 33921.5415 & 1 & 41934.5278 & 9 & 42830.6550 & 9 & 44081.4440 & 9 & 46334.5271 & 20 \\
\hline 32883.6648 & 1 & 33923.3209 & 1 & 41959.4370 & 9 & 42836.5850 & 9 & 44103.3850 & 9 & 46827.3718 & 21 \\
\hline 32911.5381 & 1 & 33927.4731 & 1 & 41960.6230 & 9 & 42836.5905 & 12 & 44167.4387 & 16 & 50096.9835 & 22 \\
\hline 32949.4949 & 1 & 33928.6591 & 1 & 41962.4024 & 9 & 42852.6010 & 12 & 44167.4388 & 16 & 50259.4818 & 23 \\
\hline 33179.6094 & 1 & 33931.6243 & 1 & 41978.4160 & 9 & 42855.5658 & 12 & 44190.5665 & 16 & 50268.3809 & 23 \\
\hline 33180.7937 & 1 & 33943.4847 & 1 & 41981.3800 & 9 & 42871.5780 & 9 & 44190.5676 & 16 & 50673.4498 & 24 \\
\hline 33183.7583 & 1 & 38652.4790 & 2 & 41982.5665 & 9 & 42947.4910 & 9 & 44278.3442 & 16 & 50839.5105 & 25 \\
\hline 33314.8284 & 1 & 38667.3120 & 2 & 41984.3449 & 9 & 43061.3603 & 9 & 44278.3443 & 16 & 50849.5929 & 25 \\
\hline 33741.8398 & 1 & 38671.4600 & 2 & 42019.3347 & 9 & 43077.3740 & 9 & 44285.4614 & 9 & 51160.3641 & 25 \\
\hline 33741.8405 & 1 & 39681.4567 & 3 & 42106.5154 & 9 & 43078.5608 & 9 & 44291.3915 & 17 & 51166.2944 & 25 \\
\hline 33761.4114 & 1 & 39776.3460 & 3 & 42304.6040 & 9 & 43115.3310 & 6 & 44345.3617 & 9 & 51179.3434 & 25 \\
\hline 33762.5979 & 1 & 39945.3718 & 4 & 42366.2810 & 10 & 43135.4945 & 9 & 44371.4559 & 9 & 51435.5490 & 26 \\
\hline 33768.5288 & 1 & 39977.3980 & 4 & 42405.4243 & 9 & 43138.4610 & 13 & 44454.4869 & 9 & 51566.6192 & 27 \\
\hline 33769.7144 & 1 & 40092.4561 & 4 & 42460.5805 & 9 & 43192.4293 & 9 & 44477.6150 & 9 & 51572.5499 & 27 \\
\hline 33769.7159 & 1 & 40127.4466 & 4 & 42465.3249 & 9 & 43198.3599 & 9 & 44582.5892 & 9 & 51577.2936 & 27 \\
\hline 33769.7174 & 1 & 40528.3615 & 5 & 42517.5142 & 11 & 43218.5236 & 9 & 44642.4879 & 16 & 51878.5755 & 26 \\
\hline 33775.6465 & 1 & 40593.5983 & 6 & 42523.4453 & 9 & 43263.5948 & 14 & 44642.4888 & 16 & 51921.2793 & 26 \\
\hline 33775.6470 & 1 & 40857.5139 & 7 & 42545.3880 & 9 & 43288.5050 & 9 & 44642.4893 & 16 & & \\
\hline 33777.4241 & 1 & 41681.2879 & 8 & 42545.3895 & 11 & 43393.4808 & 9 & 44731.4486 & 16 & & \\
\hline 33784.5405 & 1 & 41695.5194 & 9 & 42545.3909 & 11 & 43849.5518 & 9 & 44731.4491 & 16 & & \\
\hline 33791.6579 & 1 & 41697.2980 & 9 & 42603.5100 & 9 & 43878.6130 & 9 & 44731.4494 & 16 & & \\
\hline \multicolumn{12}{|l|}{ secondary } \\
\hline 44134.5281 & 16 & 44278.6418 & 16 & 44644.5686 & 16 & 50273.4290 & 23 & 51166.5939 & 25 & 51577.5847 & 27 \\
\hline 44134.5298 & 16 & 44278.6424 & 16 & 44644.5687 & 16 & 50849.2992 & 25 & 51550.3106 & 26 & 51924.5385 & 26 \\
\hline 44134.5327 & 16 & 44644.5685 & 16 & 50257.4228 & 23 & 51158.2894 & 25 & 51566.3233 & 27 & 51997.4875 & 26 \\
\hline
\end{tabular}

References: 1 - van Woerden (1957), 2 - Pohl \& Kızılırmak (1966), 3 - Kızılırmak \& Pohl (1969), 4 - Pohl \& Kızılırmak (1970), 5 - Muthsam (1972), 6 - Hilditch et al. (1979), 7 - Kızılırmak \& Pohl (1971), 8 - Kızılırmak \& Pohl (1974), 9 - Patkos (1982a), 10 - Pohl \& Kızılırmak (1975), 11 - Pohl \& Kızılırmak (1976), 12 - Mallama et al. (1977), 13 - Pohl \& Kızılırmak (1977), 14 - Mallama (1979), 15 - Pohl \& Gülmen (1981), 16 - Milano et al. (1986), 17 - Pohl et al. (1982), 18 - Braune \& Mundry (1982), 19 - Cellino et al. (1985), 20 - Pohl et al. (1987), 21 - Kundera (2001), 22 - Heckert (1996), 23 - Albayrak et al. (1996), 24 - Agerer \& Hübscher (1998), 25 - Pribulla et al. (1999), 26 - Pribulla et al. (2001), 27 - this study.

Table 3. SV Cam: third body orbit.

\begin{tabular}{lccccccc}
\hline Parameters & $\begin{array}{c}\text { Sommer } \\
(1956)\end{array}$ & $\begin{array}{c}\text { Friebos-Conde } \\
\text { \& Herczeg }(1973)\end{array}$ & $\begin{array}{c}\text { Hilditch } \\
\text { et al. (1979) }\end{array}$ & $\begin{array}{c}\text { Cellino } \\
\text { et al. (1985) }\end{array}$ & $\begin{array}{c}\text { Sarma } \\
\text { et al. (1985) }\end{array}$ & $\begin{array}{c}\text { Albayrak } \\
\text { et al. (1999) }\end{array}$ & $\begin{array}{c}\text { present } \\
\text { study }\end{array}$ \\
\hline$P_{(\mathrm{O}-\mathrm{C})(\mathrm{yr})}$ & 57.50 & 72.75 & 64.07 & 74.70 & 54.23 & 43.81 & $41.32 \pm 0.31$ \\
$A$ (days) & & & & & 0.0079 & 0.0085 & $0.0095 \pm 0.0002$ \\
$e$ & 0 & 0 & 0.60 & 0.40 & 0.59 & 0.30 & $0.29 \pm 0.03$ \\
$w\left(^{\circ}\right)$ & & 90 & 90 & 174 & 227 & $183.1 \pm 1.5$ \\
$f\left(M_{3}\right)\left(M_{\odot}\right)$ & & 0.00114 & 0.00130 & & 0.00163 & 0.00170 & $0.0030 \pm 0.0002$ \\
$M_{3}\left(M_{\odot}\right)$ & & $0.16^{a}$ & & $0.19^{a}$ & $0.18^{a}$ & $0.18^{a}$ & $0.26 \pm 0.01$ \\
\hline
\end{tabular}

${ }^{a}$ The value for $i \simeq 90^{\circ}$.

$\left(A_{\mathrm{S}}=T_{\mathrm{S}} / T_{*}\right)$, by the angular dimension (radius) of the spot $\left(\theta_{\mathrm{S}}\right)$ and by the longitude $\left(\lambda_{\mathrm{S}}\right)$ and latitude $\left(\varphi_{\mathrm{S}}\right)$ of the spot centre. The longitude $\left(\lambda_{\mathrm{S}}\right)$ is measured clockwise (as viewed from the direction of the $+Z$-axis) from the $+X$-axis (line connecting the stars' centres) in the range $0^{\circ}-360^{\circ}$. The latitude $\left(\varphi_{\mathrm{S}}\right)$ is measured from $0^{\circ}$ at the stellar equator (orbital plane) to $+90^{\circ}$ towards the "north" $(+Z)$ and $-90^{\circ}$ towards the "south" $(-Z)$ pole.

For a successful application of this model in the analysis of the observed light curves, the method of 
Djurašević (1992b) was used. Optimum model parameters are obtained through the minimization of $S=\Sigma(\mathrm{O}-\mathrm{C})^{2}$, where $\mathrm{O}-\mathrm{C}$ is the residual between the observed (LCO) and synthetic (LCC) light curves for a given orbital phase. The minimization of $S$ is performed in an iterative cycle of corrections of the model parameters. In this way the inverse-problem method gives us the estimates of system parameters and their standard errors.

We considered that Patkos's (1982a) symmetrical reference light curve is very probably clean with respect to spot effects. The maximum light level of this reference light curve coincides with the light level of the secondary maximum in our light curves. Thus we decided to normalise our light curves with respect to the secondary maximum.

The mass-ratio of the components was fixed to a revised value of $q=m_{\mathrm{c}} / m_{\mathrm{h}}=0.56$ estimated by Pojmański (1998) from the radial velocity solution. From his radial velocity study, for the mass of the primary we have $\mathcal{M}_{\mathrm{h}}\left[M_{\odot}\right] \sim 1.42$. The appropriate spectral type for this mass is F5 V, with an effective temperature $T_{\mathrm{h}}=6440 \mathrm{~K}$ (Lang 1992). This simple calibration may not work for spotted systems (in which large cool spots probably affect the spectral type determination). The light-curve analysis is made also under the assumption that primary's spectral type is close to F8 V, as suggested by Pojmański (1998). Thus we used two different values for the temperature of the hotter primary component: $T_{\mathrm{h}}=6440 \mathrm{~K}$ (Hyp. I.) and $T_{\mathrm{h}}=6200 \mathrm{~K}$ (Hip. II.) corresponding to spectral types of $\mathrm{F} 5 \mathrm{~V}$ and $\mathrm{F} 8 \mathrm{~V}$, respectively.

The values of the limb-darkening coefficients were derived from the stellar effective temperature and surface gravity, according to the given spectral type, by using the polynomial proposed by Díaz-Cordovés et al. (1995). During the process of optimisation, with the temperature changes, we have an automatic recomputation of the limb-darkening. For the $R$-filter the limb-darkening was taken from the tables of Al Naimy (1977). Following Lucy (1967), Rucinski (1969) and Rafert \& Twigg (1980), the gravity-darkening coefficients of the stars, $\beta_{\mathrm{h}, \mathrm{c}}$, and their albedos, $A_{\mathrm{h}, \mathrm{c}}$, were set at the values of 0.08 and 0.5 , respectively, appropriate for stars with convective envelopes.

Previous versions of our programme chose between two treatment of the radiation law: simple black-body theory, or stellar atmosphere models by Carbon \& Gingerich (1969) (CG). The current version of the programme (e.g. Djurašević et al. 2001) employs the Basel Stellar Library (BaSeL). We have explored the "corrected" BaSeL model flux distributions, consistent with extant empirical calibrations (Lejeune et al. 1997, 1998), and with a large range of effective temperatures $2000 \mathrm{~K} \leq T_{\text {eff }} \leq$ $35000 \mathrm{~K}$, surface gravities, $3 \leq \log g \leq 5$ and metallicity, $-1 \leq[\mathrm{Fe} / \mathrm{H}] \leq 1$, where $[\mathrm{Fe} / \mathrm{H}]$ is the logarithmic metal abundance. The surface gravities can be derived very accurately from the masses and radii of close binary $(\mathrm{CB})$ stars by solving the inverse problem of the light-curve analysis, but the temperature determination is related to the assumed metallicity and strongly depends on the photometric calibration.

In the inverse problem the fluxes are calculated in each iteration for the current values of temperatures and $\log g$ by interpolation in both of these quantities in the atmosphere tables, as an input, for a given metallicity of the $\mathrm{CB}$ components. If there is a good physical reason, the metallicity of the $\mathrm{CB}$ components could be different. But, normally, one uses the same value for both components. The two-dimensional flux interpolation in $T_{\text {eff }}$ and $\log g$ is based on the application of the bicubic spline interpolation (Press et al. 1992). This proved to be a good choice.

By selecting the particular input switch, the programme for the light-curve analysis can be simply redirected to the Planck or CG approximation, or to the more realistic BaSeL model atmospheres. The disagreements between individual $B, V$, and $R$ solutions decrease if we introduce the "corrected" BaSeL model flux distributions. A change in the assumed metallicity causes a noticeable change in the predicted stellar effective temperature. The mean value of the chemical abundances of the components was obtained by experimenting with values close to solar metallicity. For SV Cam, the best fit of the $B, V$, and $R$ light curves was obtained with $[\mathrm{Fe} / \mathrm{H}]_{\mathrm{h}, \mathrm{c}}=0.1$ for each components. With this value, the individual $B, V$, and $R$ solutions are in good agreement. The results presented here use this stellar atmosphere approximation.

In the analysis, the inclination of the orbit was estimated to be $i \sim 89.6$. The present light-curve analysis shows that during the deeper (primary) minimum the cooler (less-massive and smaller) component eclipses the hotter (more-massive and larger) one.

Since the stars in the system have external convective envelopes, which can exhibit magnetic activity, we started the "spotted solution" by assuming that the components of SV Cam have cool spots, of the same nature as solar magnetic spots. Moreover, since the results of the light-curve analysis depend on the choice of the adopted working hypothesis, the analysis was carried out within the framework of several hypotheses with spotted areas on the components.

We rejected those hypotheses which produced significantly different values of the parameters for the system and the active spotted areas, estimated by analysing the individual light curves in the $B, V$, and $R$ passbands. Finally we chose the Roche model with dark spotted areas on the more massive (hotter) component as the optimum solution. Within this hypothesis the analysis of the light curves yields mutually consistent parameters of the system and active region in the $B, V$, and $R$ passbands. In this case we obtained a very good fit to the observations.

\section{Results and discussion}

The light-time effect due to the orbit of the eclipsing pair around their center of mass with a third body has been computed with our derived parameters given in Table 3. 
Table 4. Results of the analysis of the SV Cam light curves obtained by solving the inverse problem for the Roche model with two active cool areas on the more-massive (hotter) component (Hyp. I. $-T_{\mathrm{h}}=6440$; Hyp. II. $\left.-T_{\mathrm{h}}=6200\right)$.

\begin{tabular}{|c|c|c|c|c|c|c|}
\hline Quantity & $\begin{array}{l}B-\text { filter } \\
T_{\mathrm{h}}=6440\end{array}$ & $\begin{array}{l}V-\text { filter } \\
T_{\mathrm{h}}=6440\end{array}$ & $\begin{array}{l}R-\text { filter } \\
T_{\mathrm{h}}=6440\end{array}$ & $\begin{array}{l}B-\text { filter } \\
T_{\mathrm{h}}=6200\end{array}$ & $\begin{array}{l}V-\text { filter } \\
T_{\mathrm{h}}=6200\end{array}$ & $\begin{array}{l}R-\text { filter } \\
T_{\mathrm{h}}=6200\end{array}$ \\
\hline$n$ & 242 & 242 & 242 & 242 & 242 & 242 \\
\hline$\Sigma(\mathrm{O}-\mathrm{C})^{2}$ & 0.0428 & 0.0274 & 0.0244 & 0.0432 & 0.0274 & 0.0241 \\
\hline$\sigma$ & 0.0133 & 0.0106 & 0.0100 & 0.0134 & 0.0106 & 0.0100 \\
\hline$\theta_{\mathrm{S} 1}$ & $20.3 \pm 0.4$ & $19.9 \pm 0.3$ & $19.8 \pm 0.3$ & $20.3 \pm 0.4$ & $20.2 \pm 0.3$ & $19.8 \pm 0.3$ \\
\hline$\lambda_{\mathrm{S} 1}$ & $105.3 \pm 2.4$ & $103.4 \pm 1.8$ & $111.8 \pm 1.9$ & $103.8 \pm 2.4$ & $103.7 \pm 1.7$ & $112.3 \pm 1.8$ \\
\hline$\varphi_{\mathrm{S} 1}$ & $54.7 \pm 1.1$ & $50.5 \pm 0.9$ & $50.0 \pm 1.1$ & $54.5 \pm 1.1$ & $50.7 \pm 0.9$ & $49.9 \pm 1.1$ \\
\hline$\theta_{\mathrm{S} 2}$ & $13.1 \pm 0.4$ & $14.1 \pm 0.3$ & $13.3 \pm 0.3$ & $12.7 \pm 0.4$ & $13.6 \pm 0.3$ & $12.8 \pm 0.4$ \\
\hline$\lambda_{\mathrm{S} 2}$ & $184.1 \pm 2.2$ & $185.8 \pm 1.5$ & $192.9 \pm 1.5$ & $182.7 \pm 2.4$ & $186.8 \pm 1.5$ & $195.4 \pm 1.4$ \\
\hline$\varphi_{\mathrm{S} 2}$ & $-31.1 \pm 2.6$ & $-29.3 \pm 2.0$ & $-29.8 \pm 2.8$ & $-28.4 \pm 2.8$ & $-28.6 \pm 2.4$ & $-31.3 \pm 2.8$ \\
\hline$T_{\mathrm{c}}$ & $4510 \pm 53$ & $4467 \pm 34$ & $4544 \pm 28$ & $4384 \pm 55$ & $4377 \pm 32$ & $4452 \pm 27$ \\
\hline$F_{\mathrm{h}}$ & $0.848 \pm 0.003$ & $0.848 \pm 0.002$ & $0.845 \pm 0.002$ & $0.847 \pm 0.002$ & $0.848 \pm 0.002$ & $0.845 \pm 0.002$ \\
\hline$F_{\mathrm{c}}$ & $0.712 \pm 0.002$ & $0.706 \pm 0.002$ & $0.705 \pm 0.001$ & $0.710 \pm 0.002$ & $0.707 \pm 0.002$ & $0.705 \pm 0.002$ \\
\hline$i$ & $89.6 \pm 0.9$ & $89.6 \pm 0.8$ & $89.6 \pm 0.8$ & $89.6 \pm 0.9$ & $89.6 \pm 0.8$ & $89.6 \pm 0.8$ \\
\hline$u_{\mathrm{h}}$ & 0.71 & 0.63 & 0.47 & 0.72 & 0.64 & 0.49 \\
\hline$u_{\mathrm{c}}$ & 0.82 & 0.75 & 0.65 & 0.83 & 0.76 & 0.69 \\
\hline$\Omega_{\mathrm{h}}$ & 3.443 & 3.443 & 3.453 & 3.445 & 3.442 & 3.453 \\
\hline$\Omega_{\mathrm{c}}$ & 3.743 & 3.765 & 3.770 & 3.752 & 3.764 & 3.771 \\
\hline$R_{\mathrm{h}}$ & 0.343 & 0.343 & 0.342 & 0.343 & 0.343 & 0.342 \\
\hline$R_{\mathrm{c}}$ & 0.220 & 0.218 & 0.218 & 0.219 & 0.218 & 0.218 \\
\hline$L_{\mathrm{h}} /\left(L_{\mathrm{h}}+L_{\mathrm{c}}\right)$ & 0.964 & 0.942 & 0.914 & 0.966 & 0.940 & 0.911 \\
\hline$M_{\mathrm{bol}}^{\mathrm{h}}$ & 3.62 & 3.62 & 3.63 & 3.79 & 3.79 & 3.80 \\
\hline$M_{\mathrm{bol}}^{\mathrm{c}}$ & 6.17 & 6.23 & 6.16 & 6.30 & 6.32 & 6.25 \\
\hline $\mathcal{M}_{\mathrm{h}}\left[M_{\odot}\right]$ & $1.42 \pm 0.09$ & & & & & \\
\hline $\mathcal{M}_{\mathrm{c}}\left[M_{\odot}\right]$ & $0.80 \pm 0.05$ & & & & & \\
\hline $\mathcal{R}_{\mathrm{h}}\left[R_{\odot}\right]$ & $1.38 \pm 0.02$ & & & & & \\
\hline $\mathcal{R}_{\mathrm{c}}\left[R_{\odot}\right]$ & $0.87 \pm 0.02$ & & & & & \\
\hline $\log g_{\mathrm{h}}$ & $4.31 \pm 0.02$ & & & & & \\
\hline $\log g_{\mathrm{c}}$ & $4.46 \pm 0.02$ & & & & & \\
\hline$a_{\text {orb }}\left[R_{\odot}\right]$ & $3.87 \pm 0.07$ & & & & & \\
\hline
\end{tabular}

Fixed parameters:

$[\mathrm{Fe} / \mathrm{H}]_{\mathrm{h}, \mathrm{c}}=0.1-$ accepted metallicity of the components,

$T_{\mathrm{h}}$ - temperature of the more-massive (hotter) star,

$f_{\mathrm{h}}=f_{\mathrm{c}}=1.00-$ nonsynchronous rotation coefficients of the components,

$q=m_{\mathrm{c}} / m_{\mathrm{h}}=0.56-$ mass ratio of the components,

$\beta_{\mathrm{h}, \mathrm{c}}=0.08-$ gravity-darkening coefficients of the components,

$A_{\mathrm{h}, \mathrm{c}}=0.5$ - albedo coefficients of the components,

$A_{\mathrm{S} 1,2}=T_{\mathrm{S} 1,2} / T_{\mathrm{h}}=0.65-$ spots' temperature coefficient,

BaSeL approximation of the stellar atmospheres $\left([\mathrm{Fe} / \mathrm{H}]_{\mathrm{h}, \mathrm{c}}=0.1\right.$ - accepted metallicity of the components).

Note: $n$ - number of observations, $\Sigma(\mathrm{O}-\mathrm{C})^{2}$ - final sum of squares of residuals between observed (LCO) and synthetic (LCC) light curves, $\sigma$-standard deviation of the observations, $\theta_{\mathrm{S} 1,2}, \lambda_{\mathrm{S} 1,2}$ and $\varphi_{\mathrm{S} 1,2}$ - spots' angular dimensions, longitudes and latitudes (in arc degrees), $F_{\mathrm{h}, \mathrm{c}}$ - filling factors for the critical Roche lobe of the hotter (more-massive) and cooler (less-massive) star, $T_{\mathrm{c}}$ - temperature of the less-massive cooler star, $i$ - orbit inclination (in arc degrees), $u_{\mathrm{h}, \mathrm{c}}-$ limb-darkening coefficients of the components, $\Omega_{\mathrm{h}, \mathrm{c}}-$ dimensionless surface potentials of the primary and secondary, $R_{\mathrm{h}, \mathrm{c}}$ - polar radii of the components in units of the distance between the component centres, $L_{\mathrm{h}} /\left(L_{\mathrm{h}}+L_{\mathrm{c}}\right)$ - luminosity of the hotter star (including spots on the cooler one), $M_{\mathrm{bol}}^{\mathrm{h}, \mathrm{c}} 1$ - absolute bolometric magnitudes of SV Cam components, $\mathcal{M}_{\mathrm{h}, \mathrm{c}}\left[M_{\odot}\right]-$ stellar masses in solar units, $\mathcal{R}_{\mathrm{h}, \mathrm{c}}\left[R_{\odot}\right]-$ mean radii of stars in solar units, $\log g_{\mathrm{h}, \mathrm{c}}$ - logarithm (base 10) of the mean surface acceleration (effective gravity) for system stars and $a_{\text {orb }}\left[\mathcal{R}_{\odot}\right]-$ orbital semi-major axis in units of solar radius.

The eclipsing pair completes a revolution on this orbit in $41.32 \mathrm{yr}$. The projectional angular separation between the third body and the eclipsing pair is 0 "' 19 which is within the observing limits of modern technology. We are certain that the presence of a third body in the system of SV Cam is now well established. But further observations of minima are needed for a more precise determination of the second maxima of the $(\mathrm{O}-\mathrm{C})$ variation. It is desirable to try to confirm detection by speckle interferometry which can support our position.

The parameters derived from the light curve analysis are listed in Table 4 . The errors of the parameter estimates 

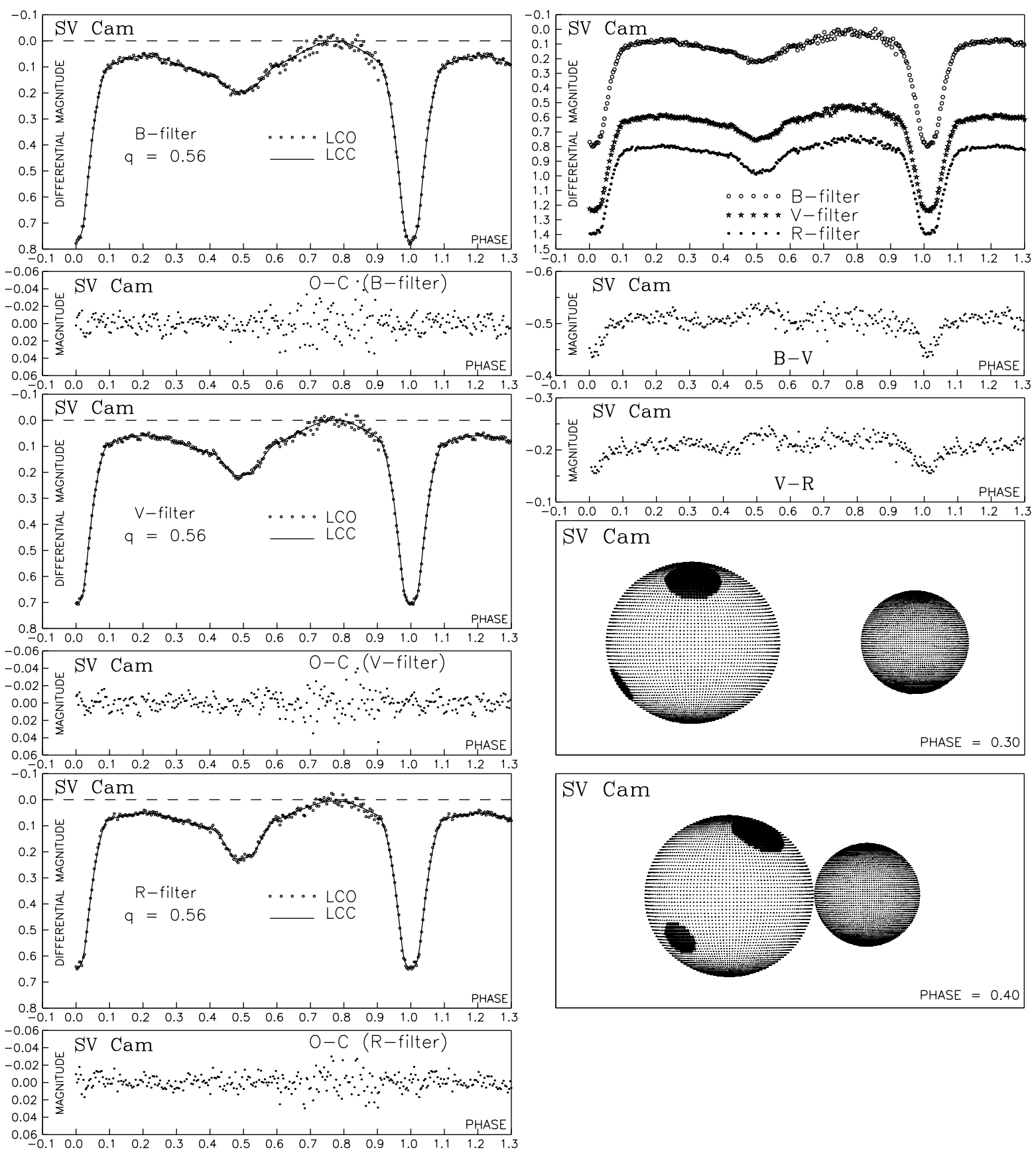

Fig. 2. Left: observed (LCO) and final synthetic (LCC) light curves of the SV Cam with final O-C residuals obtained by analysing the $B, V$ and $R$ observations; Right-top: observations of SV Cam for $B, V$ and $R$ filters respectively, and corresponding $B-V$ and $V-R$ colour indices; Right-below: the view of the Roche model for SV Cam at orbital phases 0.30 and 0.40 , obtained with parameters estimated by solving the inverse problem show both spots of the primary.

arise from the nonlinear least squares method, on which the inverse problem method is based. The fixed parameters and the meaning of the symbols in the first column are listed in a footnote to Table 4 . The spot characteristics (spot temperature factor, $A_{\mathrm{S}}=T_{\mathrm{S}} / T_{*}$; angular radius, $\theta_{\mathrm{S}}$; longitude, $\lambda_{\mathrm{S}}$; latitude, $\left.\varphi_{\mathrm{S}}\right)$ are also given in Table 4 . The determination of these parameters is based on a simultaneous fitting of the available light curves in the $B, V$ and $R$ photometric bands.
Finally, in Table 4 we provide some important absolute parameters. They were derived from the revised mass ratio of the components $\left(q=m_{\mathrm{c}} / m_{\mathrm{h}}=0.56\right)$, orbital period $\left(P=0^{\mathrm{d}} 593071\right)$, and the semimajor axis $\left(a_{\text {orb }}\left[\mathcal{R}_{\odot}\right]=3.87\right)$ found by Pojmański (1998) on the basis of the solution of the radial velocity curve. Our estimate of the accuracy in the determination of these parameters is based on the influence of formal errors arising from the nonlinear method of the light-curve analysis, and 
also on the differences between individual $B, V$ and $R$ solutions. The errors of the input parameters of the model, are treated as fixed in the inverse-problem method. They are taken from Pojmański (1998).

Using the inverse-problem solutions for individual light curves, Fig. 2 (left) presents the optimum fit of the observed light curves (LCO) by the synthetic ones (LCC). The reference light level at phase 0.75 , for the assumed unspotted configuration of the system is denoted by a dashed line. The final residuals $(\mathrm{O}-\mathrm{C})$ between the observed (LCO) and optimum synthetic (LCC) light curves are also given. A view of the Roche model of SV Cam obtained with the parameters estimated by analysing the new light curves (see Table 4) is provided in the lower right-hand panel of Fig. 2. Two cool spots were also shown on the surface of the hotter more massive primary component. We give only the graphical presentation of the results obtained within (Hyp. I.) since the differences in the fitting quality and estimated geometrical parameters of the system for both hypotheses are insignificant.

It is evident from Table 4 and on the left-hand panel of Fig. 2 that the Roche model with cool spot areas on the hotter primary component gives a satisfactory fit to the analysed new light curves. Thus the complex nature of the light curve variations of SV Cam can be explained by cool spot areas on the hotter primary component. The presence of two large cool spots leads to a conspicuous asymmetry in the light curves. Since the system's period is short $(P \simeq 0.59)$, the presence of spots at high latitudes (near the polar regions) can be explained by the dynamo mechanism for rapid rotators (Schüssler \& Solanski 1992). It is possible that large spot areas at high latitudes may correspond to an enhanced activity state of the system.

Acknowledgements. This work was supported partly by the Scientific and Thechnical Research Council of Turkey (TÜBITAK) and the Research Foundation of Çanakkale University. We appreciate the anonymous referee for her/his constructive and generous comments, Saul J. Adelman for improvements of English. We also thank the TÜBITAK National Observatory for observing time and to K. Yüce for her assistance during the observations.

\section{References}

Agerer, F., \& Hübscher, J. 1998, IBVS, No. 4606

Al Naimy, H. H. 1977, Ap\&SS, 53, 181

Albayrak, B., Özeren, F. F., \& Ekmekçi, F. 1996, IBVS, No. 4399

Albayrak, B., Özeren, F. F., Ekmekçi, F., \& Demircan, O. 1999, Rev. Mex. Astron. Astrofis., 35, 3

Braune, W., \& Mundry, E. 1982, BAV-Metteilungen, No. 34

Carbon, D., \& Gingerich, O. 1969, in Theory and Observation of Normal Stellar Atmospheres, ed. O. Gingerich (MIT Press, Cambridge), 377

Cass, T. 2000, Science, 289, 1158

Cellino, A., Scaltriti, F., \& Busso, M. 1985, A\&A, 144, 315

Díaz-Cordovés, J., Claret, A., \& Giménez, A. 1995, A\&AS, 110,329
Djurašević, G. 1992a, Ap\&SS, 196, 241

Djurašević, G. 1992b, Ap\&SS, 197, 17

Djurašević, G., Rovithis-Livaniou, H., Rovithis, P., Erkapić, S., \& Milovanović, N. 2001, A\&A, 367, 840

Friebos-Conde, H., \& Herczeg, T. J. 1973, A\&AS, 12, 11

Guthnick, P. 1929, Astron. Nachr., 235, 83

Heckert, P. A. 1996, private comminication

Hilditch, R. W., Harland, D. M., \& McLean, B. J. 1979, MNRAS, 187, 797

Kizılırmak, A., \& Pohl, E. 1969, Astron. Nachr., 292, 111

Kızılırmak, A., \& Pohl, E. 1971, IBVS, No. 530

Kizılırmak, A., \& Pohl, E. 1974, IBVS, No. 937

Kundera, T. 2001, Besançon Double and Multiple Star Database http://www.oa.uj.edu.pl/ktt-bin/wszkj.csh

Kwee, K. K., \& van Woerden, H. 1956, Bull. Astr. Inst. Neth., 12,327

Lang, K. R. 1992, Astrophysical Data: Planets and Stars (Springer-Verlag, New York Inc.)

Lejeune, T., Cuisinier, F., \& Buser, R. 1997, A\&AS, 125, 229

Lejeune, T., Cuisinier, F., \& Buser, R. 1998, A\&AS, 130, 65

Lucy, L. B. 1967, Zs. f. Ap., 65, 89

Mallama, A. D., Skillman, D. R., Pinto, P. A., \& Krobusek, B. A. 1977, IBVS, No. 1249

Mallama, A. D. 1979, IBVS, No. 1682

Marquardt, D. W. 1963, J. Soc. Ind. Appl. Math., 11, 2, 431

Mayer, P. 1990, BAIC, 41, 231

Milano, L., Mancuso, S., Vittone, A., Dorsi, A., \& Marcozzi, S. 1986, Ap\&SS, 124, 83

Muthsam, H. 1972, IBVS, No. 631

Özeren, F. F., Gunn, A. G., Doyle, J. G., \& Jevremović, D. 2001, A\&A, 366, 202

Patkos, L. 1982a, Comm. Konkoly Obs., 80, 1

Patkos, L. 1982b, in Ejection and Accretion of Matter in Binary Systems, ed. J. Tremko (Bratislava: VEDA), 61

Pohl, E., \& Kizllırmak, A. 1966, Astron. Nachr., 289, 191

Pohl, E., \& Kizllırmak, A. 1970, IBVS, No. 456

Pohl, E., \& Kizılırmak, A. 1975, IBVS, No. 1053

Pohl, E., \& Kizılırmak, A. 1976, IBVS, No. 1163

Pohl, E., \& Kizılırmak, A. 1977, IBVS, No. 1358

Pohl, E., \& Gülmen, Ö. 1981, IBVS, No. 1924

Pohl, E., Evren, S., Tümer, O., \& Sezer, C. 1982, IBVS, No. 2189

Pohl, E., Akan, M. C., İbanoğlu, C., Sezer, C., \& Güdür, N. 1987, IBVS, No. 3078

Pojmański, G. 1998, Acta Astron., 48, 711

Popper, D. M. 1996, ApJS, 106, 133

Press, W. H., Teukolsky, S. A., Vetterling, W. T., \& Flannery, B. P. 1992, Numerical Recipes in Fortran, The Art of Scientific Computing, Second Edition (Cambridge University Press, New York), 120

Pribulla, T., Chochol, D., \& Parimucha, S. 1999, IBVS, No. 4751

Pribulla, T., Vanko, M., Parimucha, S., \& Chochol, D. 2001, IBVS, No. 5056

Rafert, J. B., \& Twigg, L. W. 1980, MNRAS, 139, 78

Rucinski, S. M. 1969, Acta Astr., 19, 245

Sarma, C. V. S. R., Sarma, M. B. K., \& Abhyankar, K. D. 1985, Bull. Astr. Soc. India, 13, 346

Schüssler, M., \& Solanski, S. K. 1992, A\&A, 264, L13

Sommer, R. 1956, Astron. Nachr., 283, 155

van Woerden, H. 1957, Leiden. Ann., 21, 3

Wilson, R. E., \& Devinney, E. J. 1971, ApJ, 166, 605 